\title{
Excitation of wakefields by relativistic electron bunches in the dielectric waveguide filled with radially inhomogeneous plasma
}

\author{
G.V. Sotnikov, P.I. Markov and I.N. Onishchenko
}

NSC “Kharkov Institute of Physics and Technology”, Kharkov, Ukraine, sotnikov@kipt.kharkov.ua

One of shortcomings of the dielectric wakefield accelerators (DWA) limiting the effective length of an acceleration is susceptibility of drive bunches to beam breakup (BBU) instability [1]. With the purpose to suppress this instability recently it has been proposed to fill the drift channel of dielectric structure with plasma of certain density [2]. The investigations of plasma DWA (PDWA) [2] were made for dielectric wakefield structures with homogeneous radial plasma density. Such consideration is right if plasma comes to the drift channel from external source.

Other, widely way of plasma creation is capillary discharge. For this case on big times from the beginning of discharge (it is more than a half of pulse width of discharge current) steady distribution of plasma density with inhomogeneous distribution of discharge density forms [3]. At short distances from the axis a radial dependence of plasma density can be described with the parabolic dependence $[3,4]$

$$
n(r) \approx n_{0}\left[1+0.33(r / a)^{2}\right],
$$

where $a$ is the inner radius of dielectric tube.

Investigations on excitation of wakefield in PDWA with and inhomogeneous transverse distribution of plasma density were not carried out so far. For practical application of the proposed accelerating structure it is important to know how the accelerating gradient and focusing forces acting on test bunches will change in case when plasma in the drift channel is created as a result of the capillary discharge.

Here we report the results of numerical simulation of wakefield excitation in the dielectric structure filled with plasma created in capillary discharge. Wakefield is built up at injection of electron bunch in dielectric tube with permittivity of 3.8; outside diameter of dielectric tube (is equal to the diameter of metal waveguide) is $1.2 \mathrm{~mm}$, inner diameter is $1.0 \mathrm{~mm}$. Energy of drive bunch electrons is $5 \mathrm{GeV}$, bunch charge is $3 \mathrm{nC}$, its length was 0.2 $\mathrm{mm}$, solid bunch has diameter of $0.9 \mathrm{~mm}$. For the testing the excited wakefield we used a witness bunch. Witness bunch has the same energy and sizes as drive bunch, the charge of witness bunch was less by ten times than drive bunch charge.

The drift channel (internal area of dielectric tube) is filled with plasma of on-axis density $n_{0}=4.41 \times 10^{14} \mathrm{~cm}^{-3}$. Three different models of dependence of plasma density on radius were investigated:

a) homogeneous radial plasma density;

b) parabolic dependence of plasma density on radius according eq. (1);

c) the dependence obtained numerically by N.A. Bobrova [3] et al. when modeling capillary discharge (see Fig. 3 of Ref [3] or Fig. 2 of Ref. [4]). Last numerical dependence we will denote as BPM dependence.
At numerical simulation by means of the 2.5D PIC code created by us we examined a structure of wakefield and dynamics of electron bunches at their motion in the drift camera.

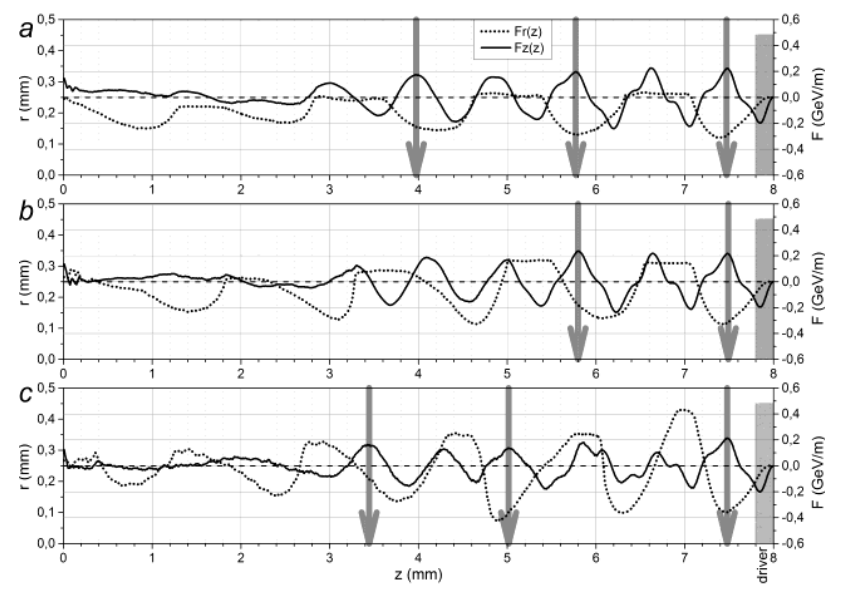

Fig. 1. Axial profiles of the transverse wakefield force (short dot line) and longitudinal wakefield force (solid line) acting on a test electron at the distance of $0.45 \mathrm{~mm}$ from center of the accelerating channel at time $t=26.69 \mathrm{ps}$ for tree cases of plasma density distributions pointed above $(\mathrm{a}, \mathrm{b}$ and $\mathrm{c})$

In Fig. 1 axial profiles of the Lorentz wakefield force acting on test electron in dielectric waveguide with plasma filling for the time $t=26.69 \mathrm{ps}$ from the beginning of injection of drive bunch for different dependences of plasma density on radius are shown: a) homogeneous, b) parabolic (1) and c) the BPM dependence [3]. The grey rectangle near output end shows the location of the drive bunch electrons. Vertical arrows have noted the possible placements of the witness bunch providing not only its acceleration at a local maximum of the longitudinal force $F_{z}$, but also the transverse focusing caused by negative value of transverse force $F_{z}$ in these locations.

One can conclude from Fig. 1 the amplitude $(\sim 200 \mathrm{MeV} / \mathrm{m})$ and frequency $(\sim 372.2 \mathrm{GHz})$ characteristics of the longitudinal wakefield (accelerating gradient) weakly depend on what model of dependence of plasma density on radius is used in calculations. At the same time the transverse (focusing) wakefield significantly changes both amplitude, and frequency characteristics. Thus for model proposed by N.A. Bobrova (Fig. 1c) amplitudes of transverse field is approximately twice more, and the frequency by 1.4 times higher, than at homogeneous distribution (Fig. 1a). It should be taken into account at elaborating the two-beam PDWA [2].

Let's consider a dynamics of drive and witness bunches in the excited wakefields. To provide the required shift of the witness bunch relative to drive bunch (see arrows in Fig. 1) it is necessary to carry out the injection of the witness bunch with certain delay $t_{d e l}$. 

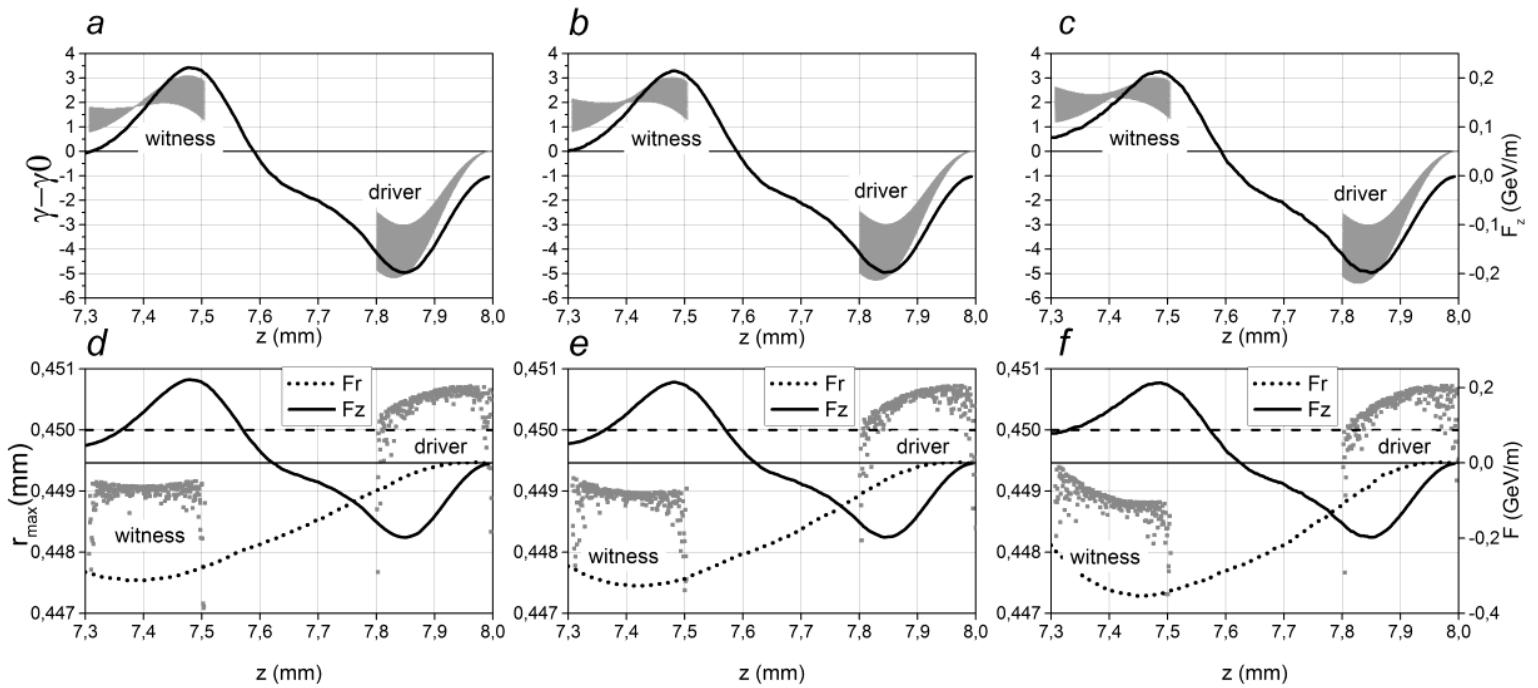

Fig. 2. a, b, c - the phase plane "energy - longitudinal coordinate" combined with dependence of longitudinal force; $d$, e, f - the configuration space displaying the positions of peripheral electrons of bunches combined with profiles of longitudinal (solid line) and transverse (dot line) forces for different dependences of plasma density on radius: $\mathrm{a}, \mathrm{d}$ - homogeneous, b, e - parabolic, c, f - the BPM dependence [3]. The delay time $t_{d e l}=1.651 \mathrm{ps}$

In Fig. 1 rightmost arrows for the three cases correspond the same delay time $t_{d e l}=1.651 \mathrm{ps}$.

At this delay it is observed both acceleration and focusing of the witness bunch for all studied dependences of plasma density on radius. The phase planes "energy longitudinal coordinate" and the positions of peripheral electrons combined with dependences of longitudinal and transverse forces for different dependences of plasma density on radius are given in Fig. 2. The left column corresponds homogeneous plasma, middle column shows the results of the simulations for the parabolic law of plasma density (1), and the right column is for the BPM dependence of radial plasma density.
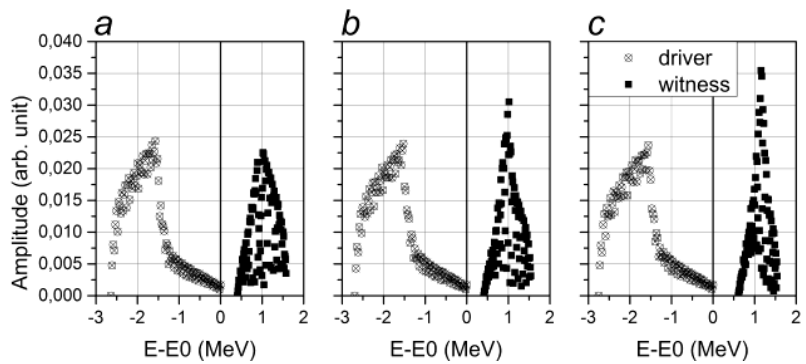

Fig. 3. Distribution functions of electrons of the drive and the witness bunches on energy at $t=26.69 \mathrm{ps}$ for different dependences of plasma density on radius: a) homogeneous, b) parabolic (1), c) the BPM dependence [3], $E_{0}=5 \mathrm{GeV}$. The delay of witness bunch is $1.651 \mathrm{ps}$

In Fig. 3 distribution functions of electrons of the drive and witness bunches on energy for different dependences of plasma density on radius at delay $t_{d e l}=1.651 \mathrm{ps}$ are shown. Comparing Figs. 2 and 3 it is possible to conclude that the greatest acceleration and focusing of test bunch are observed for the dependence of plasma density obtained numerically by N.A. Bobrova et al. [3].
If we chose other delays of witness bunch pointed in Fig. 1 by arrows we can obtain either greater acceleration or stronger focusing of witness bunch than described above. But a greater acceleration provides a worse focusing and vice versa.

The results of numerical simulation by "particle in cell" method, given above, testify that inhomogeneity of plasma in transverse cross-section is noncritical and advantages of PDWA structure with homogeneous plasma remain. I.e. even if plasma in PDWA is created by capillary discharge [3, 4], opportunity to accelerate and focus test bunch remains. Moreover, the greatest acceleration and the best focusing of test bunch are observed for the BPM dependence obtained numerically in work [3].

It should be note that the drive bunch is focused both in homogeneous, and in inhomogeneous plasma.

Work supported by NAS of Ukraine program "Perspective investigations on plasma physics, controlled thermonuclear fusion and plasma technologies", Project P-1/63-2017.

\section{References}

1. Li C., Gai W., Jing C. et al. High gradient limits due to single bunch beam breakup in a collinear dielectric wakefield accelerator // Phys. Rev. ST Accel. Beams, 17, 091302(2014).

2. Sotnikov G.V., Kniaziev R.R., Manuilenko O.V. et al. High gradient limits due to single bunch beam breakup in a collinear dielectric wakefield accelerator // Nuclear Instruments and Methods in Physics Research Section A: Accelerators, Spectrometers, Detectors and Associated Equipment. 2014, V. 740. P. 124-129.

3. Bobrova N. A., Esaulov A. A., Sakai J.-I. et al. Simulations of a hydrogen-filled capillary discharge waveguide // Phys. Rev. E, 65, 016407 (2001).

4. Steinhauer L.C., Kimura W.D. Quasistatic capillary discharge plasma model // Phys. Rev. ST Accel. Beams, 9, 081301 (2006). 